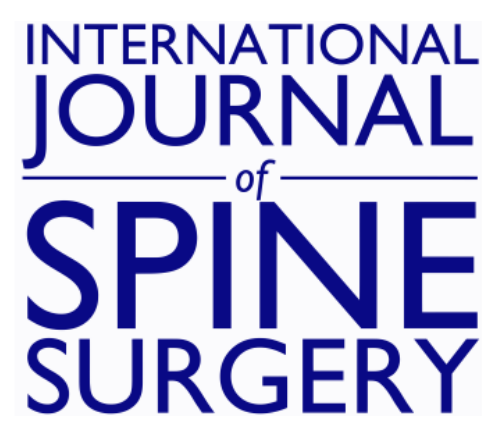

\title{
Evaluation of Spinous Process Tethering at the Proximal End of Rigid Constructs: In Vitro Range of Motion and Intradiscal Pressure at Instrumented and Adjacent Levels
}

DAVID OU-YANG, MARK MOLDAVSKY, NOLAN WESSELL, DAINA M. BROOKS, MARIANO TITANTI, ERIKA A. MATHEIS, BRANDON S. BUCKLEN and VIKAS PATEL

Int J Spine Surg 2020, 14 (4) 571-579

doi: https://doi.org/10.14444/7076

http://ijssurgery.com/content/14/4/571

This information is current as of April 26, 2023.

Email Alerts Receive free email-alerts when new articles cite this article. Sign up at: http://ijssurgery.com/alerts 


\title{
Evaluation of Spinous Process Tethering at the Proximal End of Rigid Constructs: In Vitro Range of Motion and Intradiscal Pressure at Instrumented and Adjacent Levels
}

DAVID OU-YANG, MD, ${ }^{1}$ MARK MOLDAVSKY, $\mathrm{MS},{ }^{2}$ NOLAN WESSELL, MD, ${ }^{1}$ DAINA M. BROOKS, BS, ${ }^{2}$ MARIANO TITANTI, ${ }^{3}$ ERIKA A. MATHEIS, MS, ${ }^{2}$ BRANDON S. BUCKLEN, PHD ${ }^{2}$ VIKAS PATEL, MD ${ }^{1}$

${ }^{I}$ The Spine Center at University of Colorado Hospital, Aurora, Colorado, ${ }^{2}$ Musculoskeletal Education and Research Center (MERC), A Division of Globus Medical, Inc., Audubon, Pennsylvania, ${ }^{3}$ Department of Mechanical Engineering and Mechanics, College of Engineering, Drexel University, Philadelphia, Pennsylvania

\begin{abstract}
Background: Adult spinal deformity surgery requires use of long thoracolumbar instrumentation, which is associated with risk of postoperative proximal junctional kyphosis (PJK). Tethering has been used in spinal surgery but not around the spinous process (SP) in the context of preventing PJK.

Methods: Researchers applied a nondestructive hybrid loading protocol to 7 T8-L2 cadaveric specimens in flexion-extension, lateral bending, and axial rotation (AR). A rigid construct (pedicle screws and rods) and 1- and 2-level SP constructs were tested, as was a hand-tie technique. SP tethering (SPT) constructs use clamps on both sides of the SP; SPT helix constructs use 1 clamp and wrap around the SP.

Results: All tether constructs showed greater motion at the instrumented level and less motion at adjacent levels compared to rigid constructs. In AR, 1- and 2-level SPT constructs restricted first instrumented level motion to a greater extent when compared with other tether constructs $(P \leq .05)$. Passing the band through the T10 SP did not produce significant biomechanical differences compared to passing it through the T9-T10 interspinous ligament $(P>.05)$. Hand-tied constructs demonstrated more motion compared to tensioned constructs $(P>.05)$. Intradiscal pressure results corroborated motion data.

Conclusions: SPT at the proximal end of a rigid construct produced more favorable biomechanical outcomes at instrumented and adjacent levels than were seen with a completely rigid construct. Clinical research is needed to determine whether these methods reduce the risk of PJK among patients.

Level of Evidence: 3.

Clinical Relevance: This work sheds light on the biomechanical stability of proximal tethering constructs in an effort to enhance the surgeon's ability to reduce rates of proximal junctional kyphosis and failure in thoracolumbar spinal fusion surgery.
\end{abstract}

Biomechanics

Keywords: proximal junctional kyphosis, thoracolumbar, biomechanics, hybrid testing, intradiscal pressure

\section{INTRODUCTION}

Correction for spinal deformity often requires long thoracolumbar posterior instrumentation that may cause damage to supporting soft tissue structures. Use of rigid pedicle screw and rod constructs can lead to an abrupt transition from a stiff fused segment to an unfused mobile motion segment. ${ }^{1}$ This change in motion parameters, combined with sagittal plane misalignment, may result in postoperative clinical complications such as proximal junctional kyphosis (PJK) or even proximal junctional failure (PJF). These 2 entities should be viewed on a spectrum of adjacent segment degeneration. PJK is defined by the presence of $>20^{\circ}$ of kyphosis between the upper instrumented vertebra (UIV) and the superior endplate 2 levels above. $^{2,3}$ PJK is often viewed primarily as a radiographic finding and often presents with few or only mild symptoms. ${ }^{4-7}$ PJK should be contrasted with PJF, which involves increased pain, mechanical instability, possible neurologic injury, and the need for revision surgery. ${ }^{6-8}$

The reported incidence of PJK varies considerably and can range from $17 \%$ to $39 \%$ among patients who have previously been treated for reconstruction of scoliotic deformity or kyphosis ${ }^{4}$ to $5 \%$ to $46 \%$ or even $80 \%$ within 18 months according to a recent large systematic review. ${ }^{8} \mathrm{PJF}$, 
on the other hand, occurs at a slightly more modest rate of $1.4 \%$ to $35 \% .^{7}$ It is thought to originate from high junctional stress caused by long fusion constructs, whereby a multitude of risk factors increase the probability of adjacent degeneration. Fracture at the UIV is the most common mechanism for PJK. ${ }^{4,9}$ Risk factors include low bone density, advancing age, greater number of levels instrumented (including fusion to sacrum), incorrect global spinal alignment, and sagittal vertical axis position. ${ }^{4,8,9}$ Despite the ubiquitous population affected by PJK, Kim et al, ${ }^{4}$ upon review of the literature, found no significant differences in healthrelated outcome scores; however, many patients were symptomatic and required revision surgery.

One solution that has been proposed to lessen the severity of stiffness changes associated with posterior instrumentation involves dynamic stabilization at UIVs of long fusion constructs. It is thought that junctional stresses would be reduced by gradual changes in flexibility, thus decreasing the pervasiveness of PJK. Cahill et $\mathrm{al}^{10}$ used finite element analysis to compare forces between a homogenous diameter rod and a transitional diameter rod; results showed a reduction in implant failure. Other stabilization techniques including pedicle screw, hook, and hybrid constructs have previously been studied in the literature. Results show that pedicle screw fixation significantly increases PJK, whereas hooks and hybrid constructs are more effective in decreasing proximal kyphosis. ${ }^{1-13}$

Bands and wires also may be used for posterior fixation, are available in different diameters, and consist of various materials such as stainless steel, titanium, and polyethylene terephthalate (PET). Polyethylene or polyester bands provide larger diameter stabilization and better biocompatibility when compared with metal wiring. ${ }^{14-17}$ Universal clamps with polyester bands have been used to correct deformity successfully, ${ }^{14,16,18-21}$ but the prevalence of associated PJK has not been determined. Additionally, band fixation often requires passing the bands under the lamina, which increases the risk of neurologic damage or dural injury due to positioning the band directly next to the spinal cord. The use of tethering bands around the spinous process (SP) to avoid such complications and reduce the risk of PJK, however, has not been investigated. As such, the present study aims to investigate the use of SP PET band constructs proximal to the posterior rods and screws. The authors hypothesize that SP PET reconstruction creates a gradual transfer of motion and intradiscal force at the proximal levels.

\section{MATERIAL AND METHODS}

\section{Specimen Preparation}

Seven fresh-frozen cadaveric spines were radiographed at T8-L2 in both anteroposterior and lateral planes to ensure lack of visible degeneration, osteophytes, malalignments, or instability. Specimens were then stored in double plastic bags at $-20^{\circ} \mathrm{C}$. Muscular structures and excess fat were carefully removed, while spinal ligaments, joints, and discs were preserved. Spines were fixed proximally and distally for fixation in a 3-to-1 mixture of Bondo Body Filler and fiberglass resin (Bondo/ MarHyde Corp., Atlanta, GA).

\section{Surgical Reconstruction}

We investigated motion and intradiscal pressure (IDP) of the multiple single- and multilevel SP tethering (SPT) techniques, in addition to the intact condition and pedicle screws and rods (T10-L2; rigid) constructs (Figure 1). Single-level SPT included rigid pedicle screw fixation (T11-L2) and featured: (1) T10 bilateral SPT tensioned to $275 \mathrm{~N}$, as recommended by the manufacturer (SPT 1); (2) T10 helical SPT wrapped around the T10 and T11 process and tensioned to $275 \mathrm{~N}$ (SPT Helix 1); SPT Helix 1 hand-tied (not tensioned; SPT Helix HT 1); and transhelical SPT passing through the T10-T11 interspinous ligament and tensioned to $275 \mathrm{~N}$ around the T11 SP (SPT Trans-Helix 1). Similarly, multilevel SPT included rigid fixation (T12-L2) and featured: (1) SPT 2 with bilateral SPT at T10 and T11, tensioned to $275 \mathrm{~N}$; (2) SPT Helix 2, with SPT helically tensioned around the T10, T11, and T12 processes; and (3) SPT Trans-Helix 2, with transhelical SPT passing through the T10-T11 and T11T12 interspinous ligament, tensioned to $275 \mathrm{~N}$ around the T11 and T12 processes. The proximal thoracic level instrumented with screws and rods were adjusted based on single- and multilevel tethering reconstruction such that the adjacent and first instrumented anatomic levels would be consistent throughout the study (T11 for single-level tethering, and T12 for multilevel tethering).

All constructs used standard pedicle screw fixation (REVERE Stabilization System, Globus Medical, Inc., Audubon, PA) and/or PET band 


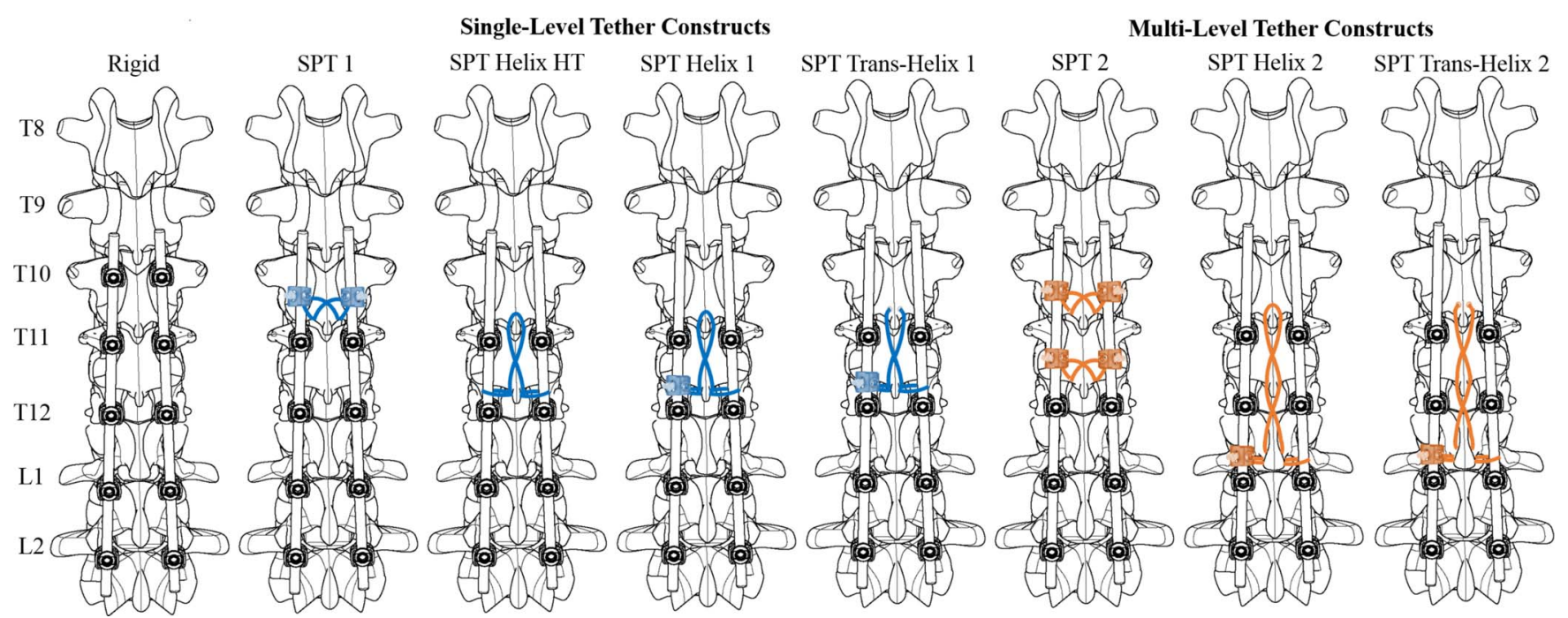

Figure 1. Schematic images depicting all 8 tether constructs (T8-L2). From left to right: (1) rigid, (2) SPT 1, (3) SPT Helix HT, (4) SPT Helix 1, (5) SPT Trans-Helix 1, (6) SPT 2, (7) SPT Helix 2, and (8) SPT Trans-Helix 2.

fixation (SILC Fixation System, Globus Medical, Inc.). The tether constructs, except for the hand-tied construct, were tensioned to $275 \mathrm{~N}$, followed by tightening of the set screw to a torque of $5.5 \mathrm{Nm}$. The band of the hand-tied construct was manually tied in a knot. Transhelix constructs required the use of a burr to create a path through the SP for passage of the tether (Figure 2).

\section{Multidirectional Testing}

Each specimen was thawed overnight and was affixed to a custom 6 degrees of freedom (6DOF) testing apparatus used to simulate physiological loads. Modeled after the spinal loading simulator proposed by Wilke et al, ${ }^{22}$ the cranial (T8) portion of the specimen was affixed to a 6DOF motor

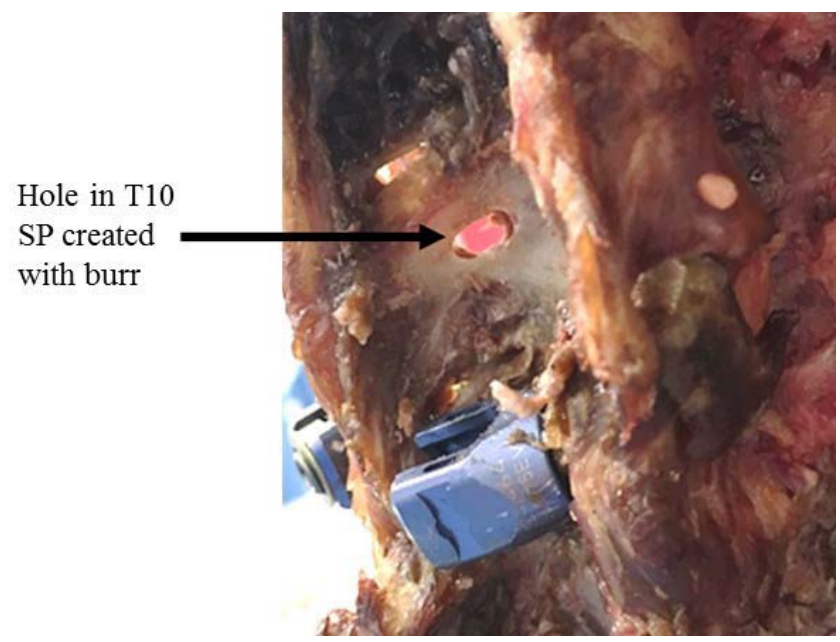

Figure 2. Photograph of interspinous ligament hole necessary for the transhelix tethering construct. gimbal assembly which applied a pure, unconstrained rotational moment independently about the $\mathrm{X}, \mathrm{Y}$, and $\mathrm{Z}$ axes corresponding to flexionextension (FE), lateral bending (LB), and axial rotation (AR). The gimbal assembly attaches to the test platform which includes linear air-bearing guide rails ( $\mathrm{X}$ and $\mathrm{Z}$ axes) and pneumatic-controlled linear actuator ( $\mathrm{Y}$ axis) enabling pure, unconstrained translation. Because the motion was unconstrained, the axis of the applied moment was constantly oriented with respect to the proximal-most vertebral level (T8). Only the caudal end of the specimen (L2) was fixed in space, as it was secured to a static, constrained testing platform. A load control protocol with servomotors will apply a pure, unconstrained bending moment, caudally, at a rate of $1.0^{\circ} /$ $\mathrm{s}$, to a maximum moment of $\pm 5 \mathrm{Nm}^{23}$ in $\mathrm{FE}, \mathrm{LB}$, and AR. Three load cycles were applied in FE, LB, and AR during quasistatic multidirectional motion testing.

Plexiglass markers, each with 3 infrared lightemitting diodes, were secured rigidly to T8, T9, T10, $\mathrm{T} 11$, and T12 vertebrae via bone screws to track motion, and the motion analysis system (Optotrak Certus, Northern Digital, Inc., Waterloo, Ontario, Canada) was placed approximately $1.8 \mathrm{~m}$ in front of the specimen. Markers denoting a rigid body were aligned along the sagittal curvature of the spine. Optotrak Certus software superimposed the coordinate systems of 2 adjacent vertebral bodies to inferentially determine relative Eulerian rotations about each of the 3 axes. At an optimal setup defined by the manufacturer, translational and 
rotational resolutions of $0.01 \mathrm{~mm}$ and $0.1^{\circ}$ and errors between $1.6 \%$ and $1.8 \%$ can be obtained. ${ }^{24,25}$ Stability of the tested constructs was assessed through measurement of peak-to-peak range of motion (ROM) reported across the adjacent level (T9-T10), first instrumented level (T10-T11), and second instrumented level (T11-T12). Data were collected during the third cycle to minimize the viscoelastic behavior of the tissues and normalized to the intact condition per specimen.

\section{Intradiscal Pressure (IDP)}

Miniature pressure transducers (Model 060, Precision Measurements Co., Ann Arbor, MI) were used to measure changes in axial loads within the adjacent level (T9-T10) and first instrumented level (T10-T11) disc spaces. The sensors used are designed for use in biomedical and biological applications with a sensing diameter of $1.5 \mathrm{~mm}$ and $0.3 \mathrm{~mm}$ thickness. Placement of the transducers within the nucleus pulposus first required a 14-gauge cannulated needle to be inserted through the anterior annulus, as recommended in literature. ${ }^{26}$ The trocar was removed, and the transducer was inserted through the cannula with the active surface facing cranially. Each transducer was placed within the center of the disc and confirmed with coronal and lateral fluoroscopy. Then the cannula was carefully withdrawn as not to disturb the placed transducer; final position of the transducers was again confirmed with fluoroscopy. Once the cannulated needle was removed, the displaced annulus fibers returned to their position, surrounding the flexible cables of the transducer. IDP was collected during FE, the motion plane that produces the largest change in hydrostatic pressure within the disc. ${ }^{26}$ All sensors were calibrated to manufacturer specifications before multidirectional testing. IDP data were acquired by using a multichannel digital acquisition device (National Instruments Corporation, Austin, TX) interfaced with a personal computer. IDP data were normalized to the intact specimen $($ intact $=100 \%)$.

\section{Data Analysis}

Statistical analysis was performed using IBM SPSS Statistics (v22, IBM Corp., Armonk, NY). Statistical analysis was performed, and ROM and IDP were compared across the proximal adjacent level (T9-T10), first instrumented level (T10-T11), and second instrumented level (T10-T11), and
Table 1. Average intradiscal pressure (IDP) in flexion-extension for the adjacent level (T9-T10) and first instrumented level (T10-T11) for all constructs. All data are normalized to intact $(100 \%)$. ${ }^{*} P \leq .05$ versus intact.

\begin{tabular}{lcc}
\hline & $\begin{array}{c}\text { Adjacent Level } \\
\text { (T9-T10) } \\
\text { Construct }\end{array}$ & $\begin{array}{c}\mathbf{1}^{\text {st }} \text { Instrumented Level } \\
\text { (T10-T11) } \\
\text { (Mean } \pm \text { SD) }\end{array}$ \\
\hline Rigid & $258 \pm 121^{*}$ & $159 \pm 71$ \\
SPT 1 & $210 \pm 87$ & $234 \pm 96^{*}$ \\
SPT Helix HT 1 & $182 \pm 76$ & $302 \pm 170^{*}$ \\
SPT Helix 1 & $181 \pm 67$ & $227 \pm 95$ \\
SPT Trans-Helix 1 & $203 \pm 74$ & $221 \pm 103$ \\
SPT 2 & $226 \pm 100$ & $230 \pm 152^{*}$ \\
SPT Helix 2 & $183 \pm 88$ & $211 \pm 152$ \\
SPT Trans-Helix 2 & $191 \pm 86$ & $207 \pm 150$ \\
\hline
\end{tabular}

grouped by single- and multilevel tethering techniques. The Shapiro-Wilk test was used to assess normality across raw ROM and IDP datasets due to the small sample size $(n=7)$. Results of the ShapiroWilk test for normality did not find any construct, across ROM and IDP datasets, to violate the assumption of normality $(P=.194-.961)$. Therefore, 1-way analysis of variance (ANOVA) with repeated measures and Tukey's post hoc analysis was used to determine significant differences in ROM and IDP between: (1) intact, rigid, SPT 1, SPT Helix 1, and SPT Trans-Helix 1 constructs; and (2) intact, rigid, SPT 2, SPT Helix 2, and SPT Trans-Helix 2 constructs. Lastly, a 1-way ANOVA with repeated measures and Tukey's post hoc analysis was used to determine the effect of tensioning or hand-tying the tethered construct on ROM and IDP by comparing the intact, rigid, and the single-level hand-tied helical construct (SPT Helix HT 1) and tensioned helical (SPT Helix 1) constructs. Statistical significance was defined as $P \leq .05$.

\section{RESULTS}

\section{Single-Level Tether Constructs}

\section{Adjacent Level (T9-T10)}

All single-level constructs (rigid, SPT 1, SPT Helix 1, and SPT Trans-Helix 1) significantly increased motion in FE and LB when compared to intact. In AR, only the rigid and SPT 1 constructs exhibited significant motion increases $(P \leq .05$; Figure 3$)$. Of IDP data recorded at the adjacent level, only the rigid construct significantly increased disc pressure compared to intact (Table 1).

\section{First Instrumented Level (T10-T11)}

The rigid construct significantly reduced motion in comparison to intact in all loading modes $(P \leq .05)$. During FE, the SPT 1, SPT Helix 1, and SPT Trans- 


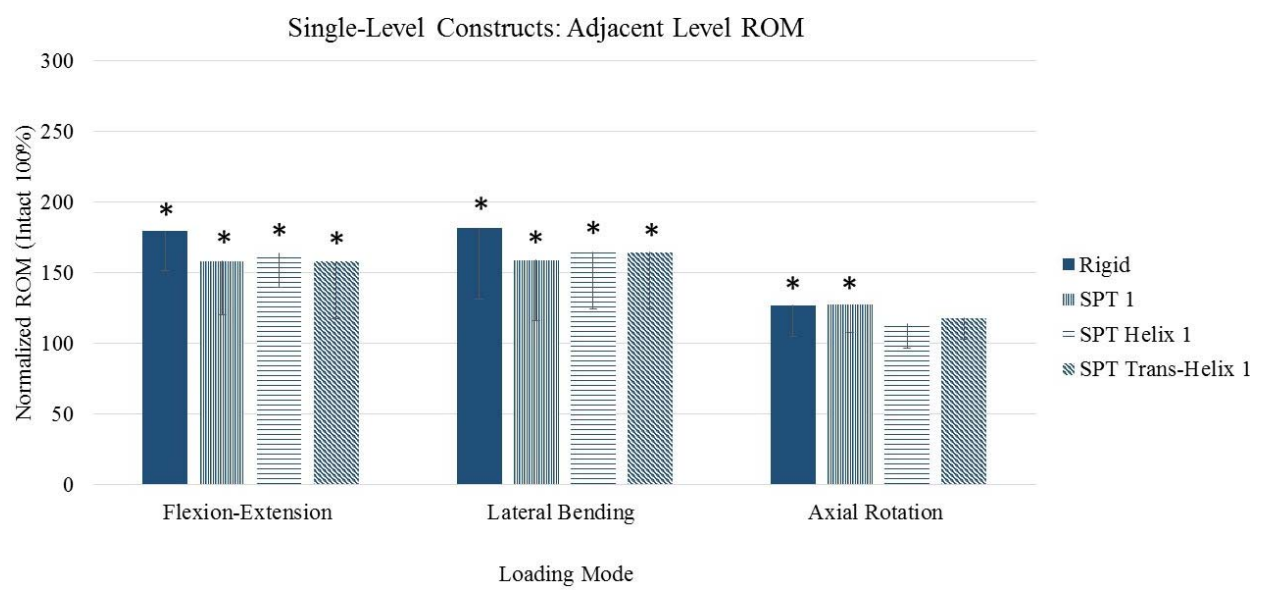

Figure 3. Average range of motion in flexion-extension, lateral bending, and axial rotation for the adjacent level (T9-T10) for single-level constructs. All data were normalized to intact $(100 \%) .{ }^{*} P \leq .05$ versus intact.

Helix 1 constructs exhibited significantly greater motion than the rigid construct $(P \leq .05)$ but showed no significant differences across groups $(P$ $>$.05). Only the SPT Helix 1 construct demonstrated increased motion in LB in comparison to the rigid construct $(P \leq .05)$. During AR, the SPT 1 construct motion was significantly less than intact $(P \leq .05)$; motion of the SPT Helix 1 and SPT Trans-Helix 1 was similar to intact $(P>.05)$ and significantly greater than rigid and SPT $1(P \leq .05$; Figure 4). Analysis of IDP data at the first instrumented level found that only the SPT 1 construct significantly increased disc pressure versus the intact spine $(P \leq .05$; Table 2$)$.

\section{Second Instrumented Level (T11-T12)}

Only the rigid construct showed a statistically significant change with a decrease in motion across all loading mechanisms versus the intact spine $(P \leq$ .05 ; Table 2).

\section{Two-Level Tether Constructs}

Adjacent Level (T9-T10)

Two-level tether constructs showed no statistically significant change in motion in any loading mechanism at the adjacent level $(P>.05$; Figure 5). No significant differences in IDP were observed (Table 2).

\section{First Instrumented Level (T10-T11)}

SPT 2 displayed increased motion in AR in comparison to the intact spine $(P \leq .05)$. The SPT 2, SPT Helix 2, and SPT Trans-Helix 2 constructs all exhibited increased motion in FE, LB, and AR in comparison to the rigid construct $(P \leq .05)$. Additionally, SPT Helix 2 and SPT Trans-Helix 2 had increased motion in AR in comparison to the SPT 2 configuration $(P \leq .05$; Figure 6$)$. FE IDP results revealed that the SPT 2 construct significantly increased pressure at the proximal instru-

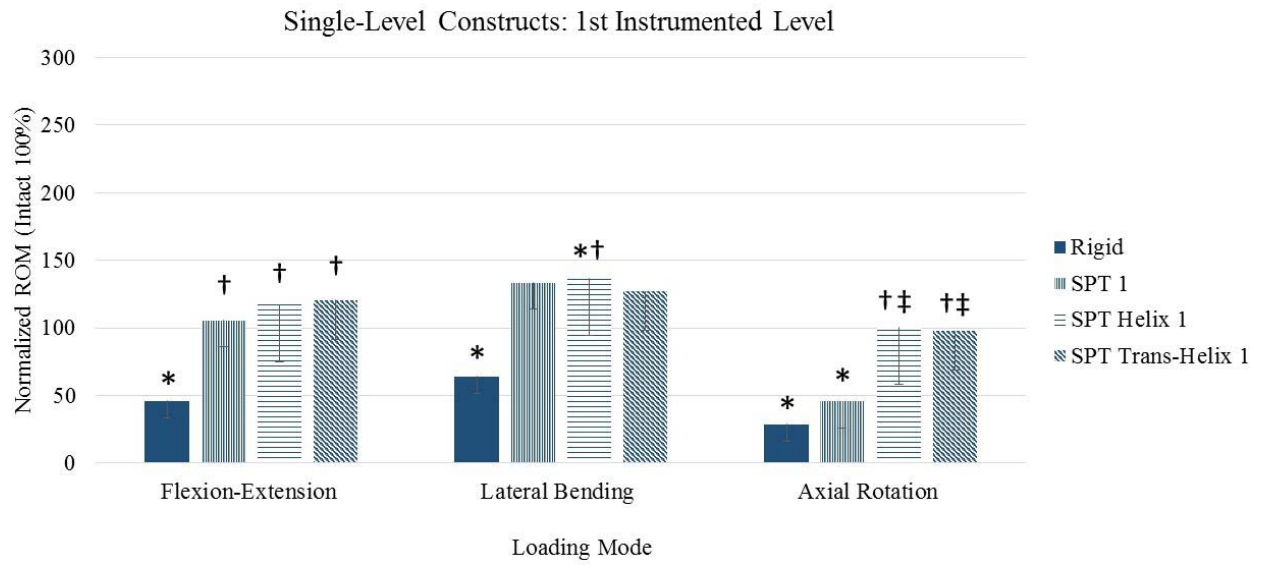

Figure 4. Average range of motion in flexion-extension, lateral bending, and axial rotation for the first instrumented level (T10-T11) for single-level constructs. All data are normalized to intact $(100 \%) .{ }^{*} P \leq .05$ versus intact; $\dagger P \leq .05$ versus rigid; $\ddagger P \leq .05$ versus SPT 1. 
Table 2. Average range of motion in flexion-extension (FE), lateral bending (LB), and axial rotation (AR) for the second instrumented level (T11-T12) for all constructs. All data are normalized to intact $(100 \%)$. ${ }^{*} P \leq .05$ versus intact; $\uparrow P \leq$ .05 versus rigid; $\ddagger P \leq .05$ versus SPT 2 .

\begin{tabular}{lccc}
\hline Construct & $\begin{array}{c}\text { FE } \\
(\text { Mean } \pm \text { SD) }\end{array}$ & $\begin{array}{c}\text { LB } \\
(\text { Mean } \pm \text { SD) }\end{array}$ & $\begin{array}{c}\text { AR } \\
(\text { Mean } \pm \text { SD) }\end{array}$ \\
\hline Rigid & $15 \pm 9^{*}$ & $20 \pm 10^{*}$ & $62 \pm 26^{*}$ \\
SPT 1 & $26 \pm 15$ & $50 \pm 21$ & $59 \pm 21$ \\
SPT Helix 1 & $33 \pm 24$ & $48 \pm 15$ & $48 \pm 18$ \\
SPT Trans-Helix 1 & $28 \pm 12$ & $52 \pm 19$ & $49 \pm 19$ \\
SPT 2 & $57 \pm 19^{* \dagger}$ & $95 \pm 24 \dagger$ & $66 \pm 23^{*}$ \\
SPT Helix 2 & $101 \pm 21 \dagger$ & $104 \pm 21 \dagger$ & $72 \pm 24$ \\
SPT Trans-Helix 2 & $91 \pm 20 \dagger \dagger$ & $103 \pm 18 \dagger$ & $74 \pm 23$ \\
\hline
\end{tabular}

mented level in comparison to intact $(P \leq .05$; Table 2).

\section{Second Instrumented Level (T11-T12)}

SPT 2 had decreased motion in FE and AR in comparison to the intact spine $(P \leq .05)$. SPT 2 , SPT Helix 2, and SPT Trans-Helix 2 displayed statistically significant increases in motion in FE and LB in comparison to the rigid construct $(P \leq$ $.05)$. Lastly, SPT Helix 2 and SPT Trans-Helix 2 had increased FE motion in comparison to the SPT 2 construct $(P \leq .05$; Table 1$)$.

\section{Hand-Tied Construct}

Adjacent Level (T9-T10)

SPT Helix HT 1 exhibited increased motion in FE and LB at the adjacent level when compared to intact $(P \leq .05)$. No significant differences in IDP were observed (Table 2).

\section{First Instrumented Level (T10-T11)}

SPT Helix HT 1 increased motion in FE and LB in comparison to both the intact and rigid constructs $(P \leq .05)$, while increased motion in AR was only seen when compared to the rigid construct $(P \leq$ .05). FE IDP for the SPT Helix HT 1 construct was largest in magnitude and was significantly greater than the intact construct at the first instrumented level $(P \leq .05$; Table 2$)$.

\section{Second Instrumented Level (T11-T12)}

There was no statistical difference in motion for any of the 3 loading modes at the second instrumented level (Table 3).

\section{DISCUSSION}

PJK is a common postoperative complication after adult spinal deformity (ASD) corrective surgery, with rates as high as $39 \% .^{4}$ PJF occurs between $1.4 \%$ and $35 \% ;^{7}$ if failure does occur, as many as $47.4 \%$ of cases require revision surgery. ${ }^{27}$ While failure modes for PJK are multifactorial, it is suggested that the abrupt transition from rigid pedicle screw fixation to the relatively hypermobile noninstrumented segments increases stress on the intervertebral discs. ${ }^{1,28}$ Consequently, posterior sublaminar tethering has been proposed to reduce the rigidity of the upper instrumented construct, in an attempt to reduce construct rigidity at the upper levels and thus minimize risk of PJK. Retrospective analysis of 625 ASD patients by Line et $\mathrm{al}^{29}$ found that prophylactic use of posterior tethering reduced rates of PJK by $20.7 \%$ compared to patients with only pedicle screws and rods $(16.1 \%$ versus $20.3 \%$, respectively); revision surgery for PJF was reduced by $61.9 \%(3.2 \%$ versus $8.4 \%)$. Nevertheless, a narrow spinal canal or risk for neurological damage may be a contraindication for sublaminar tethering. Alternatively, we propose prophylactic SPT tech-

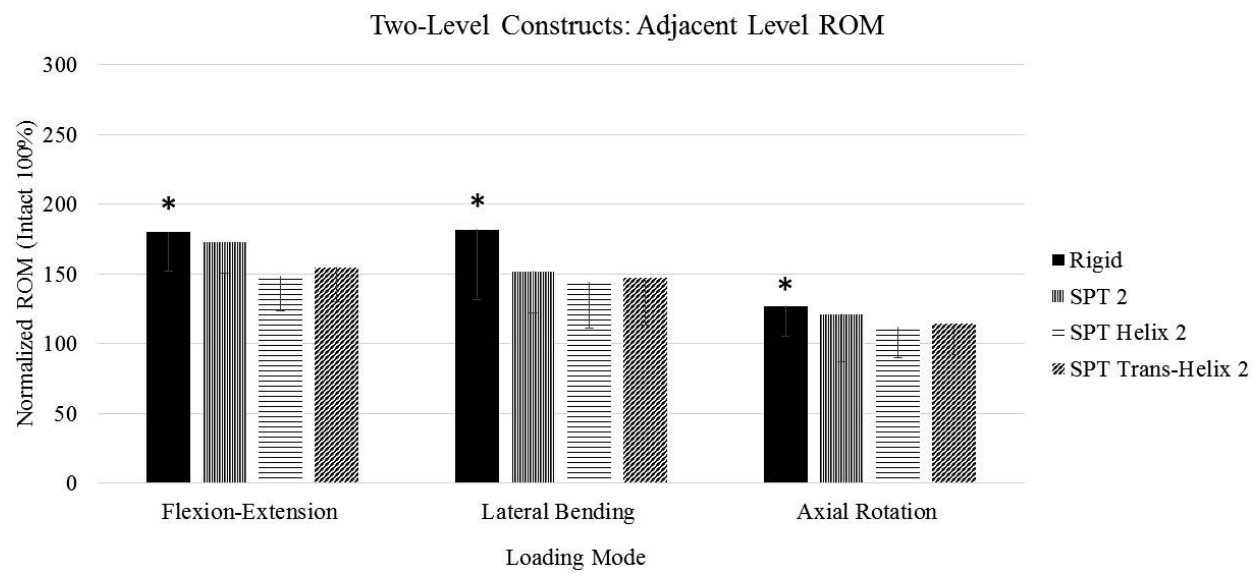

Figure 5. Average range of motion in flexion-extension, lateral bending, and axial rotation for the adjacent level (T9-T10) for 2-level constructs. All data are normalized to intact (100\%). ${ }^{*} P \leq .05$ versus intact. 
Two-Level Constructs: 1st Instrumented Level

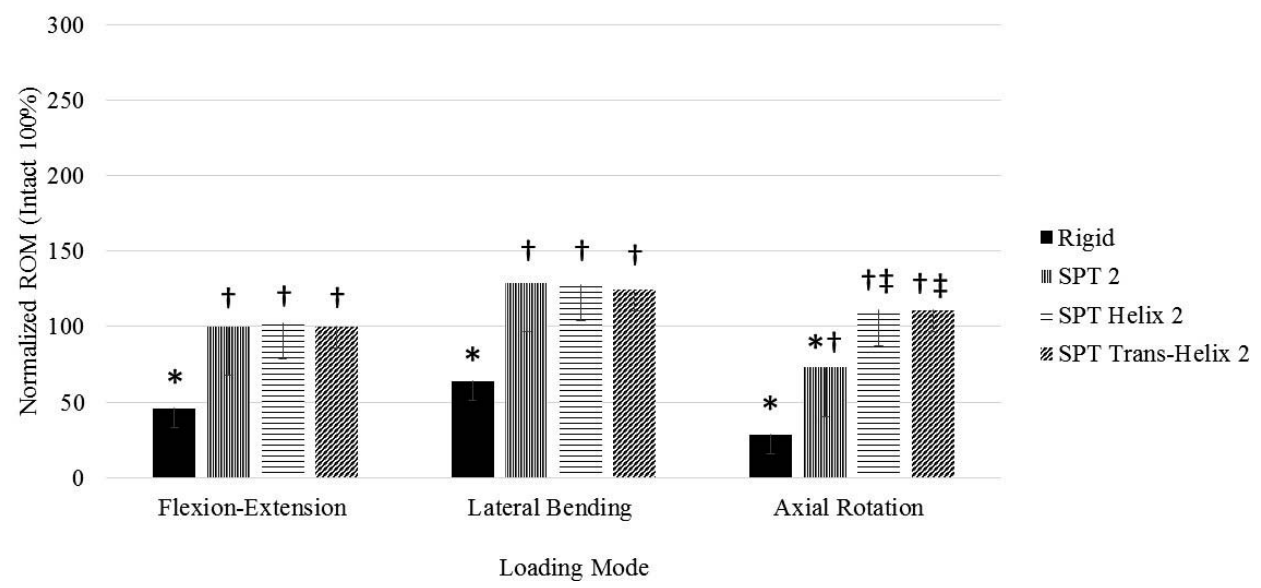

Figure 6. Average range of motion in flexion-extension, lateral bending, and axial rotation for the first instrumented level (T10-T11) for 2-level constructs. All data are normalized to intact (100\%). ${ }^{*} P \leq .05$ versus intact; $\dagger P \leq .05$ versus rigid; $\ddagger P \leq .05$ versus SPT 2.

niques and characterize the techniques compared to rigid and traditional sublaminar tethering reconstruction.

Results from the present study found that rigid construct consistently showed a significant reduction in motion across instrumented levels in every loading mode. At the adjacent level, FE IDP and average ROM were $258 \%$ and $163 \%$, respectively, in comparison to intact, reflecting the sudden transition from a stiff motion segment to an adjacent hypermobile motion segment at the proximal end of the construct. This combination of loads within the thoracic spine may be a risk factor for PJK.

Alternatively, the 1- and 2-level SPT Helix and SPT Trans-Helix constructs showed similar biomechanical effects across instrumented and adjacent levels. Investigators noted no significant difference in ROM or IDP when the tether was tensioned through the T9-T10 interspinous ligament or at the T10 SP. Contrary to SPT Helix and SPT TransHelix constructs, which require only 1 clamp and tensioning step, SPT 1 and SPT 2 constructs necessitate 2 and 4 clamps, respectively. Furthermore, clamps were placed perpendicular to the SP and in the AR plane of motion. This may explain why, during AR, SPT 1 and SPT 2 constructs showed motion trends similar to rigid constructs.

Comparisons of the tensioned and hand-tied single-level SPT Helix constructs found no significant differences in ROM and IDP between the hand-tied construct and the tensioned construct; however, the hand-tied construct did show a trend towards increased motion and pressure at the first instrumented level. This implies that, by altering tension on the tether, the surgeon may change the stiffness of the construct. However, the hand-tie technique may present the risk of intraoperative loosening of the knot over time.

There are some limitations to this study. As this was an in vitro study, it is difficult to draw conclusions about the effect of SPT in a clinical situation where bony fusion would be expected after surgery. Therefore, the authors cannot comment on how any of these configurations may alter the rate of fusion and pseudarthrosis. Also, the IDP sensors used in this study were designed to measure forces within a hydrostatic environment and are calibrated in a vacuum. Researchers inserted sensors into the T9-T10 and T10-T11 disc spaces with the goal of

Table 3. Average range of motion in flexion-extension (FE), lateral bending (LB), and axial rotation (AR) for the adjacent, first instrumented, and second instrumented levels for the hand-tied versus clamp single-level constructs. All data are normalized to intact $(100 \%)$. ${ }^{\star} P \leq .05$ versus intact; $† P \leq .05$ versus rigid; $\ddagger P \leq .05$ versus SPT 1.

\begin{tabular}{|c|c|c|c|c|c|c|c|c|c|}
\hline \multirow[b]{2}{*}{ Construct } & \multicolumn{3}{|c|}{$\begin{array}{l}\text { Adjacent Level } \\
\text { (Mean } \pm \text { SD) }\end{array}$} & \multicolumn{3}{|c|}{$\begin{array}{l}\text { First Instrumented Level } \\
\text { (Mean } \pm \text { SD) }\end{array}$} & \multicolumn{3}{|c|}{$\begin{array}{l}\text { Second Instrumented Level } \\
(\text { Mean } \pm \text { SD) }\end{array}$} \\
\hline & FE & LB & $\mathbf{A R}$ & FE & LB & $\mathbf{A R}$ & FE & LB & $\mathbf{A R}$ \\
\hline Rigid & $180 \pm 28^{*}$ & $182 \pm 50^{*}$ & $127 \pm 22^{*}$ & $46 \pm 13^{*}$ & $64 \pm 19 *$ & $29 \pm 9^{*}$ & $15 \pm 9^{*}$ & $20 \pm 10^{*}$ & $62 \pm 26^{*}$ \\
\hline SPT Helix HT 1 & $151 \pm 45^{*}$ & $161 \pm 39 *$ & $115 \pm 18$ & $147 \pm 35^{* \dagger}$ & $151 \pm 26^{* \dagger}$ & $112 \pm 22 \dagger$ & $31 \pm 27$ & $45 \pm 16$ & $49 \pm 22$ \\
\hline SPT Helix 1 & $164 \pm 24 *$ & $165 \pm 40^{*}$ & $114 \pm 17$ & $117 \pm 42 \dagger$ & $136 \pm 29^{* \dagger}$ & $100 \pm 30+\%$ & $33 \pm 24$ & $48 \pm 15$ & $48 \pm 18$ \\
\hline
\end{tabular}


placement inside the nucleus pulposus with consistent alignment from specimen to specimen. Misalignment of the sensor, degeneration of the disc, and the presence of shear forces may contribute to large standard deviations within the data. ${ }^{30-33}$ Lastly, the scope of the present study was limited to include only primary rotations in FE, LB, and AR. It should be noted that coupled motion does occur in the thoracolumbar spine, ${ }^{34}$ and future investigation should elucidate the effect of the proposed techniques on off-axis rotations.

\section{CONCLUSIONS}

Results of the current study suggest that SPT provides a gradual transfer of motion and pressure from instrumented to uninstrumented levels at the proximal end of a rigid construct. SPT constructs produced increased motion at the first instrumented level and decreased motion at the adjacent level when compared to a completely rigid construct. Hand-tying of the tether essentially allows for adjustments to the construct tension to permit more motion than would be allotted by a tensioning and clamping system but may loosen over time. These in vitro biomechanical results suggest that a new posterior fixation technique may ultimately lead to decreased risk of PJK and other complications in thoracolumbar spine fusion patients. Further studies are necessary to determine the clinical outcomes of these tethering configurations.

\section{REFERENCES}

1. Cammarata M, Aubin CE, Wang X, Mac-Thiong JM. Biomechanical risk factors for proximal junctional kyphosis: a detailed numerical analysis of surgical instrumentation variables. Spine (Phila Pa 1976). 2014;39(8):E500-507.

2. Bridwell KH, Lenke LG, Cho SK, et al. Proximal junctional kyphosis in primary adult deformity surgery: evaluation of 20 degrees as a critical angle. Neurosurgery. 2013;72(6):899-906.

3. O'Shaughnessy BA, Bridwell KH, Lenke LG, et al. Does a long-fusion "T3-sacrum" portend a worse outcome than a short-fusion "T10-sacrum" in primary surgery for adult scoliosis? Spine (Phila Pa 1976). 2012;37(10):884-890.

4. Kim HJ, Lenke LG, Shaffrey CI, Van Alstyne EM, Skelly AC. Proximal junctional kyphosis as a distinct form of adjacent segment pathology after spinal deformity surgery: a systematic review. Spine (Phila Pa 1976). 2012;37(22 Suppl):S144-164.

5. Lau D, Clark AJ, Scheer JK, et al. Proximal junctional kyphosis and failure after spinal deformity surgery: a systematic review of the literature as a background to classification development. Spine (Phila Pa 1976). 2014;39(25):2093-2102.

6. Nguyen NL, Kong CY, Hart RA. Proximal junctional kyphosis and failure-diagnosis, prevention, and treatment. Curr Rev Musculoskelet Med. 2016;9(3):299-308.

7. Cho SK, Shin JI, Kim YJ. Proximal junctional kyphosis following adult spinal deformity surgery. Eur Spine J. 2014;23(12):2726-2736.

8. Yagi $\mathrm{M}$, King $\mathrm{AB}$, Boachie-Adjei O. Incidence, risk factors, and natural course of proximal junctional kyphosis: surgical outcomes review of adult idiopathic scoliosis. Minimum 5 years of follow-up. Spine (Phila Pa 1976). 2012;37(17):1479-1489.

9. Maruo K, Ha Y, Inoue S, et al. Predictive factors for proximal junctional kyphosis in long fusions to the sacrum in adult spinal deformity. Spine (Phila Pa 1976). 2013;38(23):E14691476.

10. Cahill PJ, Wang W, Asghar J, et al. The use of a transition rod may prevent proximal junctional kyphosis in the thoracic spine after scoliosis surgery: a finite element analysis. Spine (Phila Pa 1976). 2012;37(12):E687-695.

11. Helgeson MD, Shah SA, Newton PO, et al. Evaluation of proximal junctional kyphosis in adolescent idiopathic scoliosis following pedicle screw, hook, or hybrid instrumentation. Spine (Phila Pa 1976). 2010;35(2):177-181.

12. Kim YJ, Lenke LG, Cho SK, Bridwell KH, Sides B, Blanke K. Comparative analysis of pedicle screw versus hook instrumentation in posterior spinal fusion of adolescent idiopathic scoliosis. Spine (Phila Pa 1976). 2004;29(18):2040 2048.

13. Paxinos O, Tsitsopoulos PP, Zindrick MR, et al. Evaluation of pullout strength and failure mechanism of posterior instrumentation in normal and osteopenic thoracic vertebrae. J Neurosurg Spine. 2010;13(4):469-476.

14. Ilharreborde B, Even J, Lefevre Y, et al. Hybrid constructs for tridimensional correction of the thoracic spine in adolescent idiopathic scoliosis: a comparative analysis of universal clamps versus hooks. Spine (Phila Pa 1976). 2010;35(3):306-314.

15. Ilharreborde B, Shaw MN, Berglund LJ, Zhao KD, Gay RE, An KN. Biomechanical evaluation of posterior lumbar dynamic stabilization: an in vitro comparison between Universal Clamp and Wallis systems. Eur Spine J. 2011;20(2):289-296.

16. Mazda K, Ilharreborde B, Even J, Lefevre Y, Fitoussi F, Pennecot GF. Efficacy and safety of posteromedial translation for correction of thoracic curves in adolescent idiopathic scoliosis using a new connection to the spine: the Universal Clamp. Eur Spine J. 2009;18(2):158-169.

17. Murakami H, Yamazaki K, Attallah-Wasif ES, Tsai KJ, Shimamura T, Hutton WC. A biomechanical study of 3 different types of sublaminar wire used for constructs in the thoracic spine. J Spinal Disord Tech. 2006;19(6):442-446.

18. Gazzeri R, Faiola A, Galarza M, Tamorri M. Universal Clamp system in thoracolumbar spinal fixation: technical note. Acta Neurochir (Wien). 2009;151(12):1673-1680.

19. Jouve JL, de Gauzy JS, Blondel B, Launay F, Accadbled F, Bollini G. Use of the Universal Clamp for deformity correction and as an adjunct to fusion: preliminary results in scoliosis. J Child Orthop. 2010;4(1):73-80.

20. La Rosa G, Giglio G, Oggiano L. Sagittal profile control in patients affected by neurological scoliosis using Universal Clamps: a 4-year follow-up study. Eur Spine J. 2012;21(Suppl 1):S32-36.

21. Viswanathan A, Johnson KK, Whitehead WE, Curry 
DJ, Luerssen TG, Jea A. Hybrid spinal constructs using sublaminar polyester bands in posterior instrumented fusions in children: a series of 5 cases. Neurosurgery. 2010;66(5):862867; discussion 867.

22. Wilke HJ, Wenger K, Claes L. Testing criteria for spinal implants: recommendations for the standardization of in vitro stability testing of spinal implants. Eur Spine J. 1998;7(2):148154.

23. Goel VK, Panjabi MM, Patwardhan AG, et al. Test protocols for evaluation of spinal implants. J Bone Joint Surg Am. 2006;88(Suppl 2):103-109.

24. States RA, Pappas E. Precision and repeatability of the Optotrak 3020 motion measurement system. J Med Eng Technol. 2006;30(1):11-16.

25. Milne AD, Chess DG, Johnson JA, King GJ. Accuracy of an electromagnetic tracking device: a study of the optimal range and metal interference. J Biomech. 1996;29(6):791-793.

26. Rao RD, Wang M, Singhal P, McGrady LM, Rao S. Intradiscal pressure and kinematic behavior of lumbar spine after bilateral laminotomy and laminectomy. Spine $J$. 2002;2(5):320-326.

27. Hart R, McCarthy I, O'Brien M, et al. Identification of decision criteria for revision surgery among patients with proximal junctional failure after surgical treatment of spinal deformity. Spine (Phila Pa 1976). 2013;38(19):E1223-1227.

28. Bess S, Harris JE, Turner AW, et al. The effect of posterior polyester tethers on the biomechanics of proximal junctional kyphosis: a finite element analysis. J Neurosurg Spine. 2017;26(1):125-133.

29. Line BG, Bess S, Lafage R, et al. Effective prevention of proximal junctional failure in adult spinal deformity surgery requires a combination of surgical implant prophylaxis and avoidance of sagittal alignment overcorrection. Spine (Phila Pa 1976). 2020;45(4):258-267.

30. Lee JK, Gomez J, Michelsen C, et al. In vitro biomechanical study to quantify range of motion, intradiscal pressure, and facet force of 3-level dynamic stabilization constructs with decreased stiffness. Spine (Phila Pa 1976). 2013;38(22):1913-1919.

31. Schmoelz W, Huber JF, Nydegger T, Claes L, Wilke HJ. Influence of a dynamic stabilisation system on load bearing of a bridged disc: an in vitro study of intradiscal pressure. Eur Spine J. 2006;15(8):1276-1285.

32. Stefanakis M, Luo J, Pollintine P, Dolan P, Adams MA. ISSLS Prize winner: mechanical influences in progressive intervertebral disc degeneration. Spine (Phila Pa 1976). 2014;39(17):1365-1372.

33. Weinhoffer SL, Guyer RD, Herbert M, Griffith SL. Intradiscal pressure measurements above an instrumented fusion. A cadaveric study. Spine (Phila Pa 1976). 1995;20(5):526-531.

34. Willems JM, Jull GA, J KF. An in vivo study of the primary and coupled rotations of the thoracic spine. Clin Biomech (Bristol, Avon). 1996;11(6):311-316.

Disclosures and COI: Authors VP, DO, and NW have no financial relationships to disclose. Study costs were allocated within the research budget of Globus Medical, Inc., at which MM, DMB, MT, EAM, and BSB are salaried employees.

Corresponding Author: Nolan Wessell, MD, The Spine Center at University of Colorado Hospital, 12605 E. 16th Avenue, Aurora, CO 80045. Phone: (715) 340-3715; Email: nolan. wessell@ucdenver.edu.

Published 28 August 2020

This manuscript is generously published free of charge by ISASS, the International Society for the Advancement of Spine Surgery. Copyright (C 2020 ISASS. To see more or order reprints or permissions, see http://ijssurgery.com. 